\title{
SMA-Based Muscle-Like Actuation in Biologically Inspired Robots: A State of the Art Review
}

\author{
William Coral, Claudio Rossi, Julian Colorado, Daniel Lemus and Antonio Barrientos
}

Additional information is available at the end of the chapter

http://dx.doi.org/10.5772/50209

\section{Introduction}

New actuation technology in functional or "smart" materials has opened new horizons in robotics actuation systems. Materials such as piezo-electric fiber composites, electro-active polymers and shape memory alloys (SMA) are being investigated as promising alternatives to standard servomotor technology [52]. This paper focuses on the use of SMAs for building muscle-like actuators. SMAs are extremely cheap, easily available commercially and have the advantage of working at low voltages.

The use of SMA provides a very interesting alternative to the mechanisms used by conventional actuators. SMAs allow to drastically reduce the size, weight and complexity of robotic systems. In fact, their large force-weight ratio, large life cycles, negligible volume, sensing capability and noise-free operation make possible the use of this technology for building a new class of actuation devices. Nonetheless, high power consumption and low bandwidth limit this technology for certain kind of applications. This presents a challenge that must be addressed from both materials and control perspectives in order to overcome these drawbacks. Here, the latter is tackled. It has been demonstrated that suitable control strategies and proper mechanical arrangements can dramatically improve on SMA performance, mostly in terms of actuation speed and limit cycles.

Due to their limitations, SMAs have not raised the attention of the robotics technology for several years. However, recent studies have demonstrated that by (i) finding suitable niches of application, (ii) dedicated mechatronics design, and (iii) ad-hoc control strategies, SMAs can effectively be used as an alternative actuation technology in a wide spectrum of applications and robotic systems. Indeed, as it will be introduced in this chapter, careful control design that takes into account the particular characteristics of the material coupled with proper mechanic design, play a significant role for an efficient use of SMAs. Even so, it is clear that SMAs (and smart materials in general) cannot, at this stage, be thought as a universal substitute for classical servomotor technology. However, niches of applications can be found that greatly benefit from this technology. Bio-inspired artificial systems are one such niche. 
Although SMAs are mostly used as actuators, they also have sensing capabilities. Despite most of the SMA physical parameters are strongly related in a nonlinear hysteresis fashion, the electrical resistance varies linearly with the strain of the alloy. Because strain is kinematically related to the motion of the actuator (either linear motion or rotational), the electrical resistance and the motion produced by the actuator are both linearly related. This linear relationship between resistance variation and motion is achieved because the martensite fraction is kinematically coupled to the motion, and the martensite fraction is what drives the resistance changes. This issue is an advantage for developing closed-loop position controllers that regulate the SMA actuation. In fact, most of the applications involving position linear control of SMAs, feedback electrical resistance measurements to estimate the motion generated by the actuator. This avoids the inclusion of external position sensors for closing the control loop.

SMAs are used in a variety of applications [46],[40],[56],[29],[27],[80]. Their special properties have aroused great expectations in various technologies and industries; it can be used to generate a movement or storing energy. In addition, its scope covers many sectors ranging from the use in deployable satellite antennas for different sensors to machinery, to materials for the construction of suspension bridges or anti-seismic devices. In general, all applications somehow depend on the effect of action-reaction of the material and the conditions under which particular application takes place, which make the SMAs a functional material.

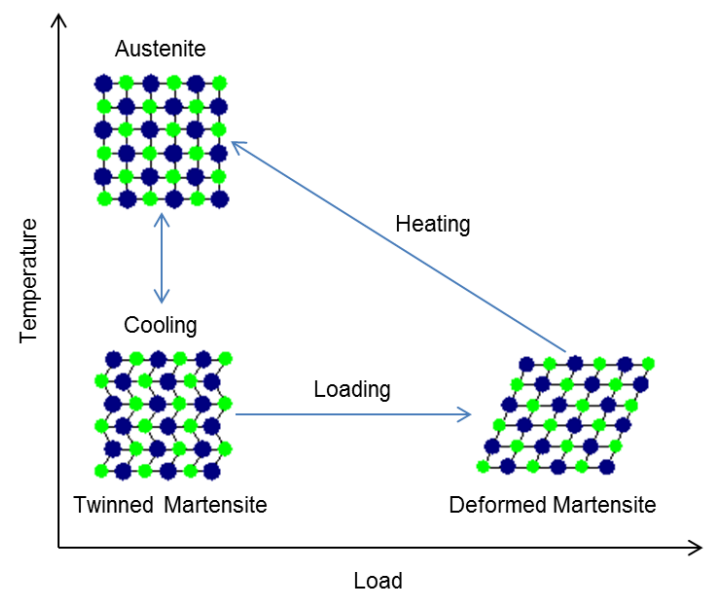

Figure 1. Microscopic viewpoint of the Shape Memory Effect

For instance, they are being used in many non-invasive surgery devices [45],[21],[62],[23],[43] and biomedicine, taking advantage of their large strains and their capability to recover the shape when the load is removed. This property allows applications in devices such as stents, tubular prosthetic devices, because it restores the ability of flow of any bodily duct affected by a narrowing.

In classical robotic systems, linear actuation systems have been proposed using SMAs. The focus of this chapter is on bio-inspired robotics. SMA-based actuators provide a suitable technology as muscle-like actuation mechanisms, which resemble the mechanics of muscles in biological systems. For this reason in the last years a number of bio-inspired robots have been 
designed adopting SMA technology. In this paper, we review the main prototypes, organizing them according to the mean (water, air, ground), and on main morphological characteristics (full body actuation or appendices only).

\section{Shape memory alloys background}

\subsection{Principle of operation}

Shape Memory Alloys are metallic materials with the ability to "remember" a determined shape, even after a severe deformation produced by a thermal stimulus. In the case of metallic alloys, the shape memory effect consists on a transition that occurs between two solid phases, one of low temperature or martensitic and other of high temperature or austenitic. The material is deformed in the martensitic phase and retrieves, reversibly, its original dimensions by heating above a critical transition temperature. The terms martensite and austenite originally referred only to the steel phases, however these terms have been extended referring not only to the material but also the kind of transformation. Thereby, the martensite steel involves a change of volume and shape, while the SMA has basically a change of length.

In general, NiTi (Nickel-Titanium) SMAs are the most common alloys used. This is basically because these materials are intrinsically susceptible of use both as sensors and actuators, which makes them suitable for integration in smart structures. NiTi SMAs work based on the shape memory effect, which essentially takes place by the influence of temperature change of the material; i.e. the temperatures at which the martensitic and austenite phase transformations begin and end. Figure 1 depicts how these changes occur at the microscopic level of the material. The phase transition occurs when the material is heated or cooled. In general, there is a certain temperature range for the transition, which is mainly defined by the manufacturer.

SMAs normally exhibit one-way shape memory effect, also called memory effect in a simple manner. The alloy deforms upon heating but cooling does not change the shape unless it is stressed again. The percentage of deformation of NiTi alloys (\% of strain) is about five percent, a range considerably higher if one considers that the deformation of common steel allows only an average of two percent. Currently, SMAs that exhibit two-way shape memory effect are also manufactured. In this case, the alloy expands by heating above the range of transition temperature and spontaneously contract when cooled again below this temperature [47]. To produce the double shape memory effect, the material is subjected to heat treatment, also called training. This training-phase forces the material to remember both heating and cooling states.

From the microscopic viewpoint (Figure 1), all the physical properties of the alloy vary depending on the phase, i.e. from cooling to heating and vice versa. Some of these properties refer to corrosion resistance, elasticity, damping capacity, strain, stress, electrical resistance, and temperature. Therefore, shape memory alloys behave in a thermo-mechanical way, with all these variables strongly coupled within a nonlinear hysteresis fashion.

Table 1 shows the commercial characteristics of SMAs depending on the diameter of the wires (NiTiNol ${ }^{\circledR}$ ). From the table it can be noticed their high electrical power consumption. In robotics applications, power consumption is a critical issue due to the level autonomy of the robotic system is fully dependent on the capacity of the onboard batteries. 


\begin{tabular}{c|c|c|c|c|c}
\hline $\begin{array}{c}\text { Diameter Size } \\
\text { inches }(\mathrm{mm})\end{array}$ & Resistance & $\begin{array}{c}\text { Pull Force } \\
\text { pounds } \\
\text { (grams) }\end{array}$ & $\begin{array}{c}\text { Approximate } \\
\text { Current for 1 } \\
\text { Second } \\
\text { Contraction } \\
(m A)\end{array}$ & $\begin{array}{c}\text { Cooling Time } \\
158^{\circ} \text { F,70 C } \\
\text { "LT" Wire } \\
\text { (seconds) }\end{array}$ & $\begin{array}{c}\text { Cooling Time } \\
194^{\circ} F, 90^{\circ} \mathrm{C} \\
\text { "HT" Wire } \\
\text { (seconds) }\end{array}$ \\
\hline \hline $0.001(0.025)$ & $36.2(1425)$ & $0.02(8.9)$ & 45 & 0.18 & 0.15 \\
\hline $0.0015(0.038)$ & $22.6(890)$ & $0.04(20)$ & 55 & 0.24 & 0.2 \\
\hline $0.002(0.050)$ & $12.7(500)$ & $0.08(36)$ & 85 & 0.4 & 0.3 \\
\hline $0.003(0.076)$ & $5.9(232)$ & $0.18(80)$ & 150 & 0.8 & 0.7 \\
\hline $0.004(0.10)$ & $3.2(126)$ & $0.31(143)$ & 200 & 1.1 & 0.9 \\
\hline $0.005(0.13)$ & $1.9(75)$ & $0.49(223)$ & 320 & 1.6 & 1.4 \\
\hline $0.006(0.15)$ & $1.4(55)$ & $0.71(321)$ & 410 & 2 & 1.7 \\
\hline $0.008(0.20)$ & $0.74(29)$ & $1.26(570)$ & 660 & 3.2 & 2.7 \\
\hline $0.010(0.25)$ & $0.47(18.5)$ & $1.96(891)$ & 1050 & 5.4 & 4.5 \\
\hline $0.012(0.31)$ & $0.31(12.2)$ & $2.83(1280)$ & 1500 & 8.1 & 6.8 \\
\hline $0.015(0.38)$ & $0.21(8.3)$ & $4.42(2250)$ & 2250 & 10.5 & 8.8 \\
\hline $0.020(0.51)$ & $0.11(4.3)$ & $7.85(3560)$ & 4000 & 16.8 & 14 \\
\hline
\end{tabular}

Table 1. Characteristics of NiTinol®SMA wires [6].

\subsection{Improving the performance of SMA actuators}

One of the main limitations in SMA actuation speed is due to high latency that the the cooling time of the wire implies. Despite increasing the input heating power can reduce the heating time, large cooling times limit the operation frequency of the actuator. On average, NiTi wires with a diameter of $127 \mu \mathrm{m}$ typically requires an electrical current input about $320 \mathrm{~mA}$ to contract in about $1 s$ (nominal heating time) and relax in approximately 1.4s (nominal cooling time). In this case both contraction and recovery times would set a nominal actuation frequency about $0.416 \mathrm{~Hz}$, quite slow for many applications requirements.

Research to overcome this limitation has been oriented towards developing cooling systems for SMAs, aimed at decreasing the nominal cooling time involved during the recovery process. In this direction, temperature control methods have been proposed in [41]. Cooling systems based on Peltier cells [18] or active cooling [67], have been commonly used. However, nowadays bio-inspired robotic systems tend to be small and light, therefore other methodologies for enhancing SMA actuation speed must be addressed. For several years different strategies have been proposed to implement rapid control in the SMA wires $[12],[19],[70],[72],[11],[71],[75]$. A system consisting of rapid heating of the SMA was proposed by [12] aimed at increasing the overall actuation frequency by means of overloading the operation of SMAs. The term overloading refers to increasing the amount of input heating power to be delivered to the SMA wires. In [74] experiments carried out using a two degree-of-freedom Pantograph robot actuated by an antagonistic pair of SMA wires acting 
as linear actuators have shown how the nominal actuation frequency was increased from $0.416 \mathrm{~Hz}$ to $1 \mathrm{~Hz}$.

Overloading should be monitored in order to avoid overheating problems that may cause physical damage of the shape memory effect. In [71], further research in this direction allowed for the introduction of a force control architecture with the proper mechanisms for safe overload the operation of SMA actuators. In the prototypes described in Section 4, we have used a control architecture similar to the one described in [71], which makes use of proper mechanisms to overload the operation of SMAs. However, these mechanisms have been adapted to work within a position control scheme, avoiding the need of including external force sensors. Section 4.2 will detail on this issue.

Besides rapid heating techniques to overload SMA operation, further investigations have been also carried out to verify whether SMAs can respond to high frequencies. In [64] and [73] experiments have demonstrated that NiTi SMA wires with a diameter of $0.1 \mathrm{~mm}$ can respond up to frequencies of $2 \mathrm{KHz}$. This high-frequency response corresponds to small-signal heating currents inputs with frequencies of that magnitude. These results allow for the development of small-signal high-bandwidth controllers capable of improving SMA performance, but more important, eliminating the limit cycles of operation of SMAs. In other approaches, $20-30 \mathrm{~Hz}$ limit cycles have been observed, whereas in [19], [50] at approximately $100-200 \mathrm{~Hz}$. In this regard, the use of high-bandwidth force sensors might be suitable for developing a SMA force feedback control system.

\subsection{Modeling and control}

The physical behavior of SMAs is more complex than many common materials: the stress-strain relationship is nonlinear, hysteresis is presented, large reversible strains are exhibited, and it is temperature dependent. This thermo-mechanical relationship can be described by formulating phenomenological models. Tanaka in [69] was one of the pioneers to study a stress-induced martensite phase transformation, proposing an unified one-dimensional phenomenological model that makes use of three state variables to describe this process: temperature, strain, and martensite fraction. His main contribution was to demonstrate that the rate of stress is a function of strain, temperature and martensite fraction rates. Later, Elahinia [7], [8] proposed an enhanced phenomenological model compared to other works [40], [31], [69] and also addressed the nonlinear control problem. This model was able to better describe the behavior of SMAs in cases where the temperature and stress states changed simultaneously. Their model was verified against experimental data regarding a SMA-actuated robotic arm [10].

Phenomenological models may provide some insights of SMA thermo-mechanical behavior that facilitate the development of control procedures. To control purposes, parameters' tuning is highly dependent of a modeling stage, but definitively phenomenological models are not the best choice for control design, especially if the goal is related to improving actuation speed. In this direction system identification is a promising alternative. As noted by [40], [19], [11], [71], [20], identified linear models for SMA can be developed. It has been demonstrated that the AC response of NiTi SMA wires behave as a first order low-pass filter. 
Section 4 of this paper details two different approaches for modeling SMAs; one based on identifying how electrical resistance change as a function of the input current [59], and the other based on identifying how the output torque produced by an antagonistic pair of SMA actuators change as as a function of the applied power [5]. Furthermore, [5] details how to take advantage of phenomenological models for simulating overheating problems when a SMA wires are overloaded. Attempting to perform this analysis on the real SMA actuators might cause several damage to the structure. Phenomenological models are really useful for determining the upper limits of applied input heating currents.

The control methods presented in [59] and [5] have been conceived for controlling a pair of antagonist SMA actuators. The antagonistic configuration is useful for having SMA actuators where each direction of motion can be controlled. In [59], the antagonistic pair of actuators must bend the structure of the fish robot, whereas in [5] the antagonistic SMA actuators are connected to a joint for providing the rotational motion. Other approaches in [11], [30], [41], [28] have demonstrated the advantages of using an antagonistic arrangement in terms of controllability. When the active actuator is being heated while the passive (antagonistic) is cooling, hysteresis effects are reduced due to the external stress that the active actuator applies on the inactive one above the austenite finish temperature.

\section{Bio-inspired robots with SMA muscle-like actuation}

The use of SMAs as artificial muscles allows for more realistic bio-inspired actuation presented in nature [26]. SMA wires acting as muscle fibers can respond upon electrical signals, taking advantage of the large pull force and its excellent strength-weight tradeoff. Currently, the use of SMAs in biomimetic robotic systems [3],[13],[9] can be found in ground, water and air robots, in many sizes, including those micro-robots or microstructures $[25],[78],[53],[35],[63],[82],[37],[37]$. In the following sections, we describe the most representative bio-inspired robots and structures that integrate SMAs as muscle-like actuation mechanisms.

\subsection{Water}

Biologically inspired robots that operate in water can be found in two categories. Firstly, robots that use SMAs for actuating appendices (fins), and secondly, robots that use SMAs to actuate the robot's body. In the latter body actuation is used for undulatory motion (fish-like robots). Some animals can move by bending their body in such a way to produce a backward-propagating propulsive wave. The movement obtained by bending a continuous structure is much more natural than others where joints are presented. In Section 4.2 our bending structure prototype is presented.

\subsubsection{A micro-robot fish with embedded SMA wire actuated by flexible biomimetic fin}

In [78] it has been proposed a micro robot fish that uses a flexible biomimetic fin propeller with embedded SMA wires to mimic the musculature and flexible bending of squid fin. The propulsion consists of an active component (the biomimetic fin) and a passive component (the caudal fin). The biomimetic fin-based propulsion mechanism is an actuator that combines the SMA wire and an elastic substrate. 
This micro-robot fish introduces a new concept in the world of biomimetic robotics due to its ability to swim noiseless. This means the robot avoid the use of any traditional components like gears, bearings and joints, only using the SMAs as actuators that produce the propulsion. The robot is able to achieve a swimming speed of $112 \mathrm{~mm} / \mathrm{s}$ when the SMAs actuate at $2.1 \mathrm{~Hz}$ (contracting upon electrical heating), and a minimum turning radius of $136 \mathrm{~mm}$, which makes the robot the fastest micro robot-fish compared to other prototypes that use IPMCs like actuators [14],[54],[33]. The authors have measured the robot performance based on the Strouhal number [15], [24], which typically varies between 0.25 to 0.35 for the biological counterparts. Their robot has a Strouhal number of 0.58 (at maximum swimming speed). This upper value highlights the optimal movement of the robot, however, high amounts of input power have been required to actuate the SMAs.

\subsubsection{Towards a biologically inspired small-scale water jumping robot}

In [63], the locomotion description of a water-jumping robot that mimic the ability of the water striders and the fishing spider to jump on the water surface. This biomimetic robot achieves a vertical jumping motion by pushing the water surface. The motion is triggered with a latch driven by the SMA actuator.

As a result of the research, quantification of $\operatorname{Re}=260$ (Reynolds number is the ratio of inertial over viscous forces), $\mathrm{Bo}=0.0054$ (Bond number is the ratio of the buoyancy to the surface tension) and $\mathrm{We}=4.7$ (Weber number is the ratio of the inertia to the surface tension) and the $B_{a}$ (Baudoin number is the ratio of the body weight to the surface tension) suggest that the physics of jumping in this robot is similar to those of the fishing spider. The Bond, Weber number and Baudoin numbers are explained by [15], [24]. In terms of actuation, the SMA allows the robot to be extremely light (mass of $0.51 \mathrm{~g}$ ), which it is essential to ensure the buoyancy on water. The maximum jumping height is $26 \mathrm{~mm}, 26 \%$ of the height reached when jumping on ground $(53.1 \mathrm{~mm})$. This prototype is the first concept of jumping robot that integrates SMAs within a structure with an overall mass of $1 g$. The robot requires $2 W$ of power consumption in order to generate a force of $1.35 \mathrm{mN}$.

\subsubsection{A micro biomimetic manta ray robot fish actuated by $S M A$}

In [79] a manta ray robot fish actuated by SMA wires is designed. Figure 2 shows the prototype of the robot. Two pectoral fins arranged in triangular-shaped made of latex with a thickness of $0.2 \mathrm{~mm}$ form the fin surface.

This micro manta ray was the first prototype that uses SMAs to generate thrust. This robot is capable to swim forward and turn. The sweep back angle of the pectoral fins is $20^{\circ}$. A maximum swimming speed of $57 \mathrm{~mm} / \mathrm{s}$ was achieved and the maximum amplitude of the motion was $40 \mathrm{~mm}$. All the biomimetic fins are open-loop controlled.

\subsubsection{Controlling a lamprey-based robot with an electronic nervous system}

In [81] a sea Lamprey has been developed. The robot consists of a cylindrical electronics bay propelled by an undulatory body axis. SMA actuators generate propagating flexion waves in five undulatory segments of a polyurethane strip. The lamprey robot Figure 3(a) consists on a cylindrical hull that houses the electronics and battery pack. In this application, the authors use a neuronal network that allows the robot to be controlled in real time. This neuronal 


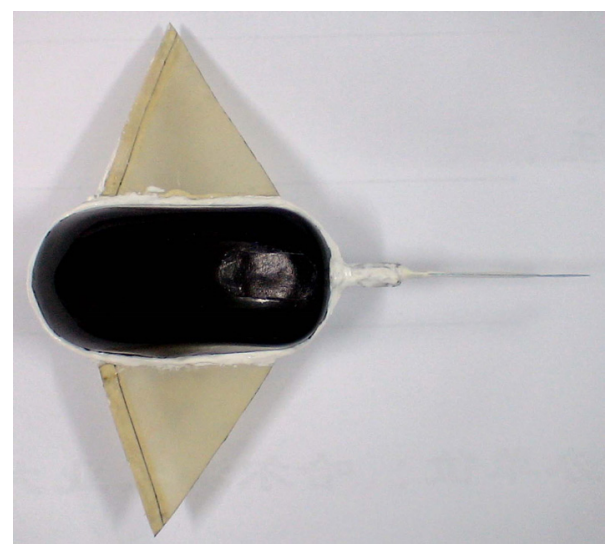

Figure 2. Micro biomimetic manta ray robot fish [79].

network also drives the control of the SMAs. The results have shown the system can reject disturbances thanks to the robustness of the nonlinear controller [55]. Each SMA wire drains $1.5 \mathrm{~A}$ of electrical current when activated.

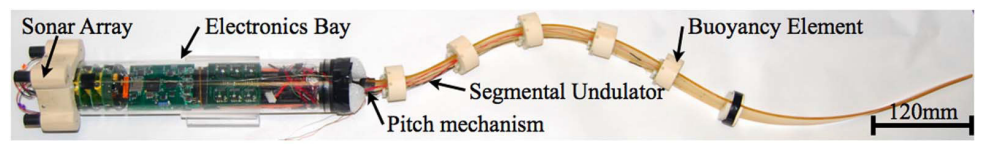

(a)

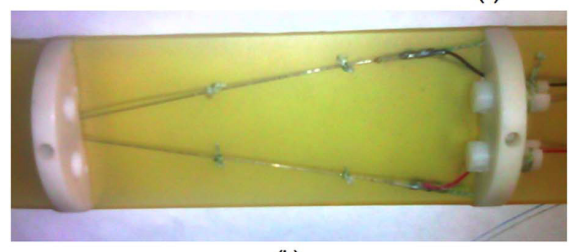

(b)

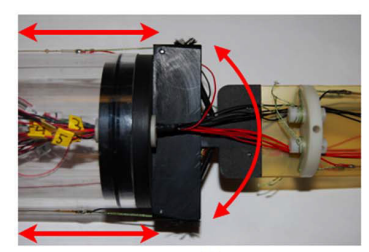

(c)

Figure 3. (a) Lamprey Robot with sonar array, (b) Lateral view of tail segment showing nitinol actuator, Teflon vertebra and tensioning nuts and (c) Lateral view of pitch mechanism [81].

\subsubsection{A biomimetic robotic jellyfish (Robojelly) actuated by shape memory alloy composite actuators}

The newest and more advanced aquatic robot that uses SMA actuators is a jellyfish robot designed by [68]. The hydrogen-fuel-powered robot called "Robojelly" mimics the propulsion, morphology, kinematics and physical appearance of a medusa (jellyfish); the Aurelia aurita species. The bio-inspired actuators are made of silicone, SMA wires and spring steel.

The development of Robojelly has introduced a systematic method for the design and fabrication of SMA-based actuators called BISMAC (bio-inspired shape memory alloy composite). This method allows for bending the structure of the robot by means of SMA contraction [77]. Thanks to the BISMAC SMA arrangement, this robot was capable to mimic the physics and swimming characteristics of jellyfish in terms of A. aurita's bell geometry, passive relaxation mechanism, neutral buoyancy, frequency of motion, and 
deformation-to-flap motion profiles. The structure can be bended by the SMAs actuators (deformation), and then a flap motion of the bell-segment structures provide the propulsion. The Robojelly was able to produce enough thrust to propel itself and achieve a proficiency of $0.19 s-1$ which is comparable to the natural medusa at $0.25 s-1$. The robot consumes an average of $16.74 \mathrm{~W}$ over its 14 th cycle of actuation. This robot confirms the fact that most aquatic biomimetic robots use SMA wires combined with other materials to create SMA-based actuators. This characteristic shows the flexibility of the SMA to work in combination with other materials.

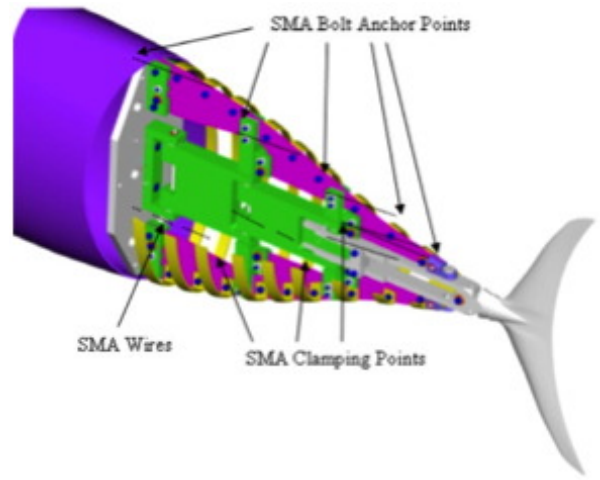

Figure 4. A CAD representation of the tail of Suleman's Tuna [65].

\subsubsection{Design and testing of a biomimetic tuna using shape memory alloy induced propulsion}

In [65], an SMA actuated tail inspired by a Bluefin tuna is proposed. Figure 4 shows the tail cutaway. This fish-like robot has length of $1 \mathrm{~m}$ capable of the biological carangiform swimming mode. The maximum tail beat frequency was $0.5 \mathrm{~Hz}$ due to the limitations of the SMA. Even at this low frequency, the power requirements were significant. The minimum and maximum power consumptions were calculated to be $292.8 \mathrm{~W}$ and $333.6 \mathrm{~W}$ respectively.

\subsection{Air}

In aerial bio-inspired robots most of the applications are appendices. Here, we can identify two main categories: insects and birds. To the best of the authors' knowledge only one robotic flying insect has been developed, apart form the jumping robot described earlier. This can be explained by the flapping frequency needed, far form the SMA's capabilities, and also by their power requirements. For these reasons insect-like flying robots mostly adopt piezo-electric actuators. In fact, the flying insect prototype described below uses SMA to fold and unfold the wings, and not for the primary flapping motion. Despite SMA actuation speed does not allow the actuation of flapping wings, it could allow for other kind of wing actuation, such as morphing-wings.

\subsubsection{Recent progress in developing a beetle-mimicking flapping-wing system}

In [51] a beetle-like insect robot inspired by the Allomyrina Dichotomapresents is presented. This robot features a morphing-wing airfoil capable of folding and unfolding the hind wing 
using SMA wires. A single small size DC motor drives the flapping mechanism. Figure 5 shows the prototype and the unfolding of the artificial flapping/morphing wing device.

Similar folding ratio of the robot's wings has been observed in comparison with the biological counterpart, accounting for 1.7 of value. On average, wing unfolding was completed within about $3 s$ and the wing folded in about $4 s$.
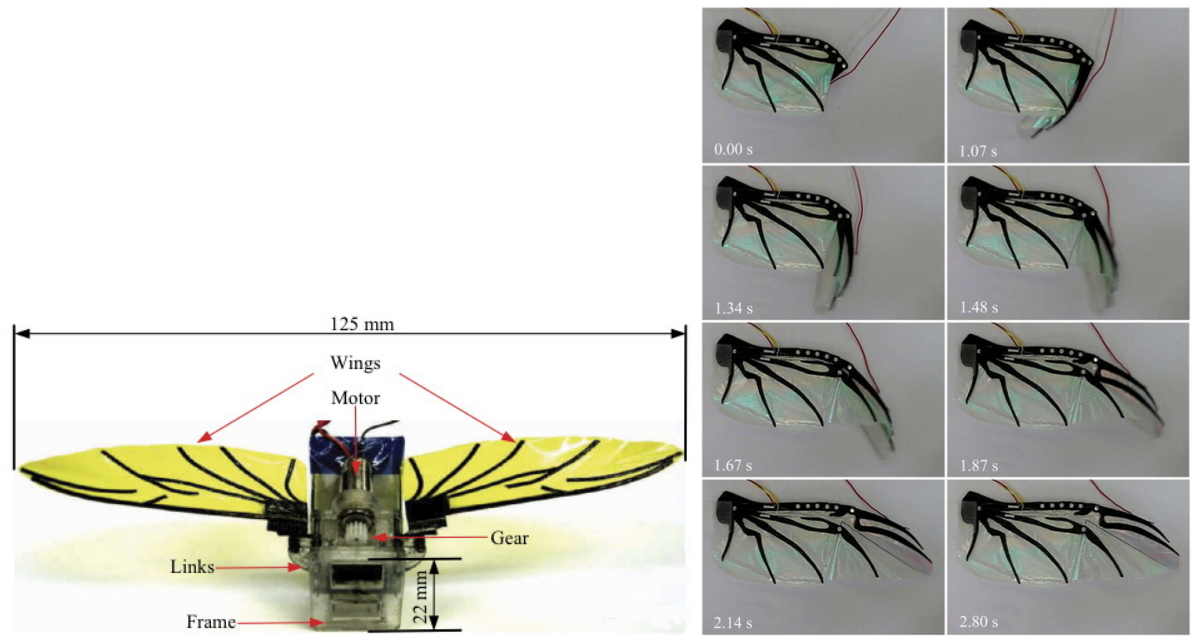

Figure 5. Prototype of the robotic beetle and detail of the unfolding of the artificial wing [51].

\subsubsection{BATMAV-a biologically inspired micro-air vehicle for flapping flight: artificial-muscle based actuation}

The BATMAV is a biologically inspired bat-like Micro-Aerial Vehicle (MAV) with flexible and foldable wings capable of flapping flight [2]. The robot features bat-inspired wings with a large number of flexible joints that allow mimicking the kinematics of a real bat flyer. Figure 6 details the overall structure of the robot, and the main connections of the SMA-like muscles.

BATMAV is the first robot that uses the SMA wires to play a dual role: first, as muscle-like actuators that provide the flapping and morphing wingbeat motions of the robot, and second, as super-elastic flexible hinges that join the wing's bone structure. Most of the experiments in [? ] were carried out with a two-degree of freedom wing capable of flapping at $3 \mathrm{~Hz}$. Despite the fact that their robot is able to achieve accurate bio-inspired trajectories, the results presented lack experimental evidence of aerodynamics measurements that might demonstrate the viability of their proposed design.

\subsection{Ground}

Ground bio-inspired robots have been divided in two categories: the ones that uses actuated appendices (i.e, legged robots) and those that actuate the whole body, i.e. crawling robots such as snakes and worms. 


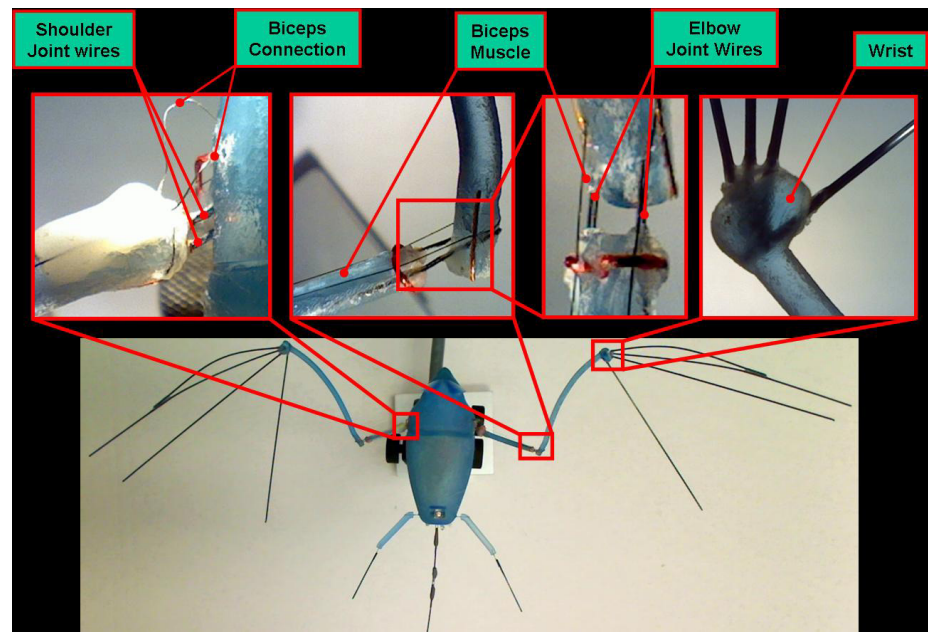

Figure 6. BATMAV. Dual Role of Shape Memory Alloy wires: as actuation muscles, and super elastic joints. (Picture from https://sites.google.com/site/gheorghebunget/research/batmav, with permission).

\subsubsection{Sensor fusion in a SMA-based hexapod bio-mimetic robot}

In [44] SMABOT is presented, a hexapod biomimetic robot with two SMA actuators that allow for the motion of the two degree-of-freedom robot. Each SMA actuator produces 300 gram - force of pull force. Figure 7 shows the SMABOT IV. SMABOT IV incorporates two-dimensional inertial navigation system for position control. The average speed when moving with tripod gait is $30 \mathrm{~cm} / \mathrm{min}$. Its maximum power consumption is about $25 \mathrm{~W}$ (the mass is $290 g$ ).

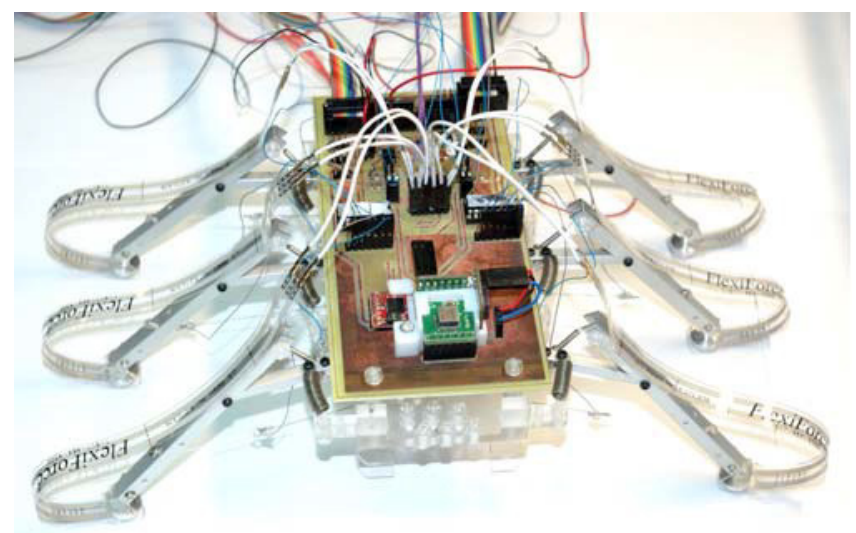

Figure 7. SMABOT IV, a SMA based hexapod robot with the IMU module, compass sensor and step touch sensors [44]. 


\subsubsection{Omegabot: Crawling robot inspired by Ascotis Selenaria}

In [38] a robot inspired by the inchworm Ascotis Selenaria is presented. The robot, called Omegabot, is named after the omega $(\Omega)$ shape of the crawling motion of the inchworm. Figure 8 shows the Omegabot platform. Previous work about this robot can be also found in [37].

Experimental results report the first step for establishing an inchworm-like robot that can crawl on various terrains where conventional robots cannot move. The Omegabot uses a SMA coil actuator that requires a current of $200 \mathrm{~mA}$ for activation. The frequency of motion is about $1 \mathrm{~Hz}$, limited by the response time of the SMA wires. The inchworm robot is manually controlled by an IR remote operation, and it achieves a maximum linear velocity of $5 \mathrm{~mm} / \mathrm{s}$. The robot travels a distance of $5 \mathrm{~mm}$ per stroke.

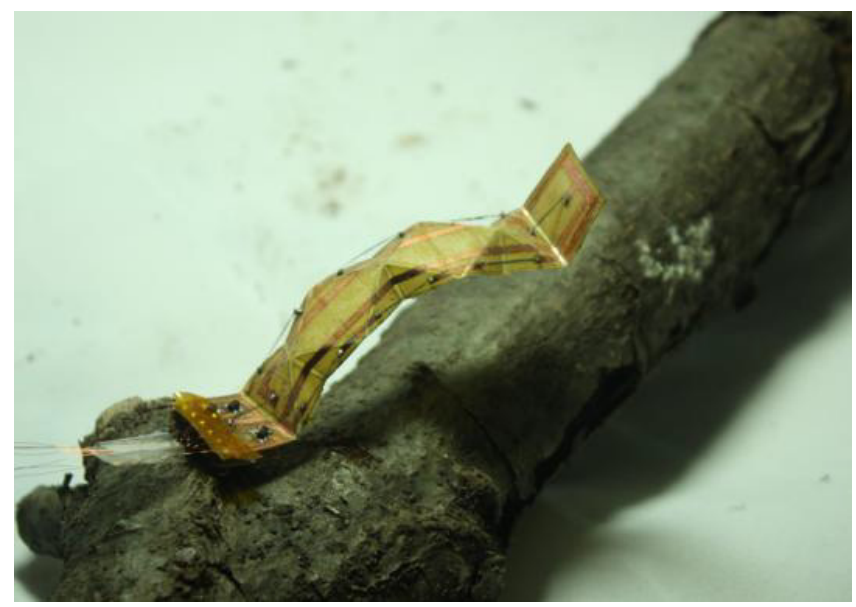

Figure 8. Omegabot, a biomimetic inchworm robot, grasps the branch of a wood, raises its head, and turns right. Bottom right: Proleg of Omegabot [38].

\subsubsection{An earthworm-like micro robot using shape memory alloy actuator}

In [34] a bio-mimetic micro earthworm-like robot with wireless control is proposed. The actuation mechanism consists on a SMA spring that contract and extend the earthworm muscle respectively. The proposed mechanism is simple but effective when traveling in narrow and rough environments, such as human digestive organs, bended long pipeline and so on. Also, this micro robot incorporates both control and power supply onboard. The theoretical speed of the micro robot is approximately $3.4 \mathrm{~mm} /$ cycle, where the total time per cycle is $8 s$ (the contraction time of the SMA is $2 s$, whereas the recovery time is $6 s$ ). The fabricated micro robot can move with the velocity of $10 \mathrm{~mm} / \mathrm{min}$ during 8 minutes. The stroke per cycle is $2.0 \mathrm{~mm}$.

\subsubsection{Other peristaltic motion concepts}

A concept similar to the one depicted in Figure 9 has been proposed in [48]. A SMA-spring has been used for changing the axial length of worm's modules, and consequently changing 
their width. The robot has a length and diameter of $1 \mathrm{~cm}$. SMA springs of 50 and $100 \mu \mathrm{m}$ wires have been tested. The resulting prototype, made of 4 modules, was able to achieve a speed up to $0.22 \mathrm{~mm} / \mathrm{sec}$, with a power consumption of $600 \mathrm{~mA}$. The previous prototype was composed of a number of identical segments attached in series, each of which alternately contract axially and expand radially. To conclude this section two prototypes are worth mentioning that use SMAs structures for peristaltic motion.

The proposed structure in these cases is a tubular mesh made of SMA wires that convert radial contraction into longitudinal lengthening. Such a structure has been proposed independently in [61] (see also [36]) and in the SoftWorm project [4].
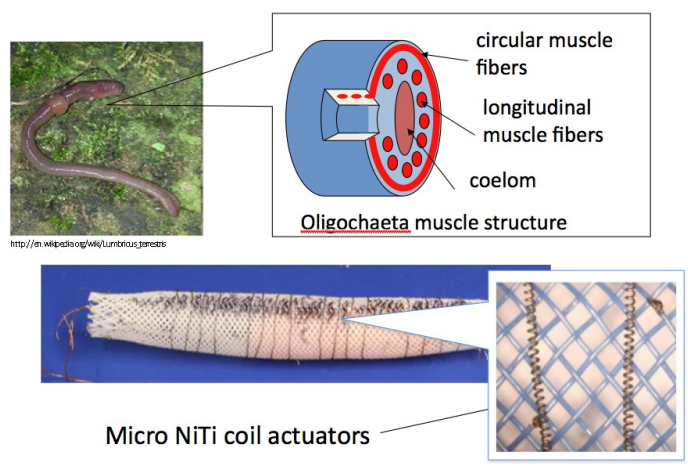

Figure 9. Arrangement of Antagonistic circular muscles in Oligochaeta [48] (Image of the real Oligochaeta from http://en.wikipedia.org/wiki/Lumbricus_terrestris).

\subsection{Other SMA-based actuation systems}

In this section we present two works that do not address the development a full robot, but rather studying and developing appendices to be added to future full robotic systems.

\subsubsection{Research on Development of a Flexible Pectoral Fin Using Shape Memory Alloys}

In [83], experimental research on pectoral fin structure is presented. The design of the pectoral fin actuator is based on SMAs wires composed by a couple of plates with the opposite functions. Figure 10 shows the biomimetic pectoral fin.

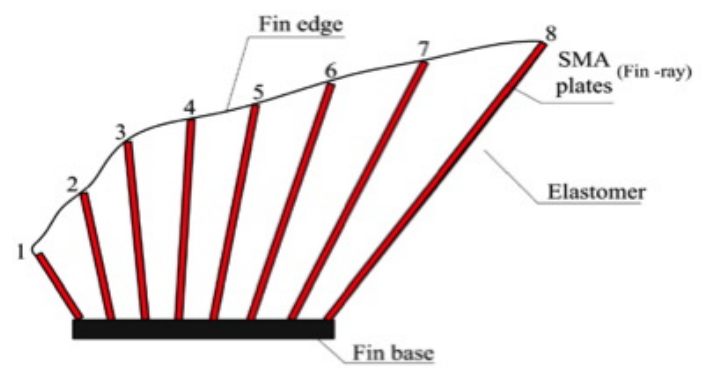

Figure 10. Biomimetic pectoral fin driven by eight couples of SMA plates [83]. 
This pectoral fin was the first and today continues being the only designed with only SMA wires. In fact, most research on fish-like robots is focused on studying propulsion (how to generate thrust), while maneuvers is largely unexplored.

\subsubsection{Development of a dexterous tentacle-like manipulator using SMA-actuated hydrostats}

Novel design principles and technologies for a new generation of high dexterity soft-bodied robots inspired by the morphology and behavior of the octopus are being developed in the framework of the OCTOPUS-IP project ${ }^{1}$.

The imitation of the internal muscular structure of octopuses' tentacles is being studies and imitated. Longitudinal cables and transverse SMA imitate the arrangement of muscle fibers, controlling contractions as soft actuators within the robot arm [42]. Moreover, this manipulator is surrounded by a sensitive skin, with contact sensors embedded into silicone rubber, equipped with passive suckers that allow the grasping of objects. SMA actuators are used to change the section of the tentacle in several locations, inducing its bending.

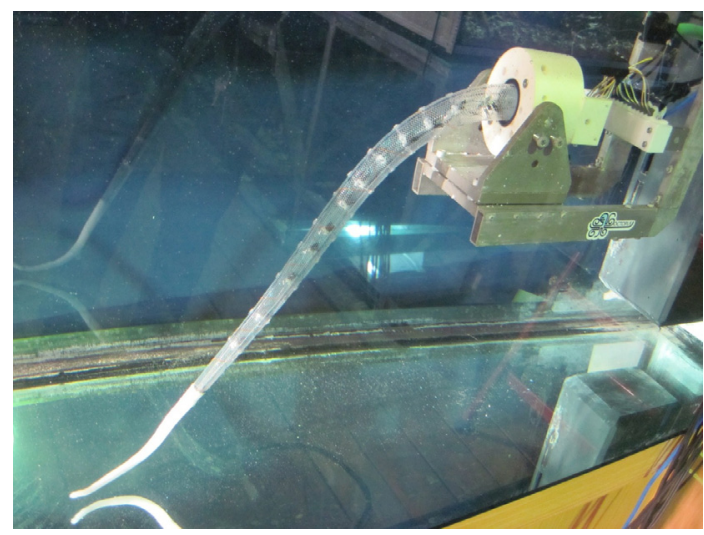

Figure 11. The SMA-based tentacle (See http://www. octopusproject.eu/).

\subsubsection{Development of a Shape-Memory-Alloy actuated biomimetic hydrofoil}

The development and testing of a biomimetic active hydrofoil using Shape Memory Alloy (SMA) actuators is presented in [16]. This work describes the development and testing of a six-segment demonstration foil and the control schemes used.

\section{Review on recent advances: iTuna and BaTboT}

In this section, we report our most recent results on two SMA-actuated bio-inspired robots. The first, called iTuna, ia an underwater robot that according to our classification falls into the "full-actuated-boy" category. The second is an aerial robot, which implements the concept of morphing wings by means of SMA-based muscles.

\footnotetext{
${ }^{1}$ http://www.octopusproject.eu/
} 


\section{1. iTuna: a bending structure swimming robotic fish}

The iTuna [59] is a swimming fish-like robot that apart from the external appearance, imitates some key features of the internal morphology of fishes.

This mechatronic concept takes inspiration from the arrangement of the red or slow-twitch muscles (see inset in Figure 12). In live fishes, such muscles are used for bending a flexible but nearly incompressible axis. Such axis is either composed of a (visco) elastic beam (notochord) or a series of vertebrae connected through intervertebral discs. The main structure of the iTuna robot fish is inspired by the former solution, and is composed by a continuous flexible backbone. The backbone is composed of polycarbonate of $1 \mathrm{~mm}$ thickness actuated by SMA muscles acting as red muscles.

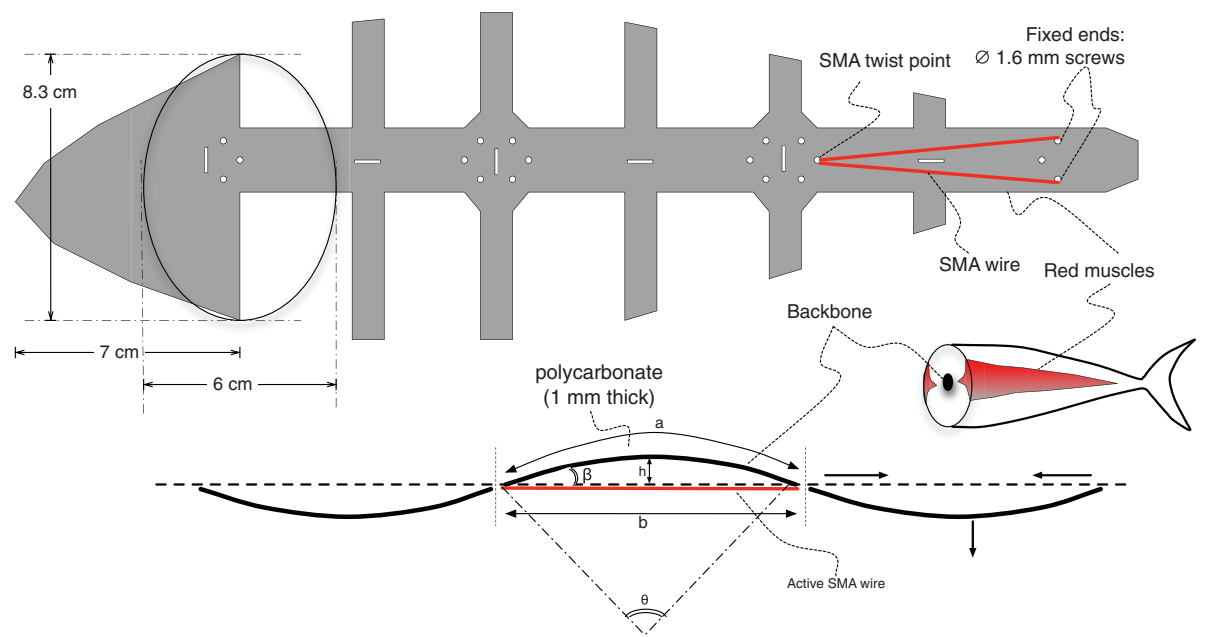

Figure 12. Main structure of the iTuna robot fish. $\mathrm{a}=8.5 \mathrm{~cm}$. Under nominal operatrion, $b \cong 96 \% \mathrm{a}=8.16$ $\mathrm{cm}, \mathrm{h}=1.02 \mathrm{~cm}, \mathrm{~b}=28^{\circ}$ [59].

Six SMA-based actuators whose length is $1 / 3$ of the body length are positioned in pairs, parallel to the body in such a way to produce an antagonistic movement on three body segments of $8.5 \mathrm{~cm}$ length. This antagonistic configuration of SMA wires has some advantages in terms of increasing the range of controllable actuation, since both directions of motion (contraction and elongation) can be actively controlled. Figure 12 shows the location of the SMA wires within the skeleton structure of the prototype.

A V-shaped configuration of the wires, where each artificial muscle is composed of a single V-shaped SMA wire, twisted around the tension screw, allows to double the pull force without a significant increase of power consumption. NiTi SMA wires with a diameter size of $150 \mu \mathrm{m}$ have been adopted. These have a pull force of 230 grams - force at consumption of $250 \mathrm{~mA}$ at room temperature, and a nominal contraction time of 1 second.

Under nominal operation such SMAs can bend the body segments up to 28 degrees (angle $\beta$ of Figure 12), even if SMA wires only contract approximately $4 \%$ of their length. By increasing the input electrical current and including a suitable control that handles an overloaded SMA operation, contraction time of $0.5 \mathrm{~s}$ was achieved, and strain could be increased up to $6 \%$, corresponding to a bending of approximately $36^{\circ}$ (Fig. 13). 


\subsubsection{SMA control in the iTuna}

After identification, a low-level PID controller has been designed to address two main limitations of SMAs: slack in the fibers, and limited actuation speed. Slack issues appear when SMA wires develop a two-way memory effect during operation [11]. Limitation in actuation speed occurs due to the large switching time between cooling and heating phases. To address such problems, a pre-heating mechanism has been developed that works in conjunction to the antagonistic arrangement. The pre-heating avoids the temperature on both wires drops below the $10 \%$ of the maximum applied electrical current, preventing the inactive alloy from complete cooling. On the other hand, the antagonistic arrangement provides an external stress to the cooling wire (provided both by the elastic backbone and by the active antagonistic wire). Working with an already-warm wire allows for a faster stretch and slack issues are avoided. Note that the PID controller is based on the experimental observation that the hysteresis on the electrical resistance curve was smaller than the hysteresis on the temperature curve. Resistance measurements are used as a feedback signal for closed-loop control (see [59] and [60] form more details).

The control developed allows overloading the SMA with up to $350 \mathrm{~mA}$ peak current (note that power signals are sinusoidal, hence overloading only lasts a brief period of time). Overloading has allowed for achieving a $1 \mathrm{~Hz}$ oscillation time (i.e. 0.5 seconds contraction and cooling times) and a bending angle of 36 degrees of each body segment.

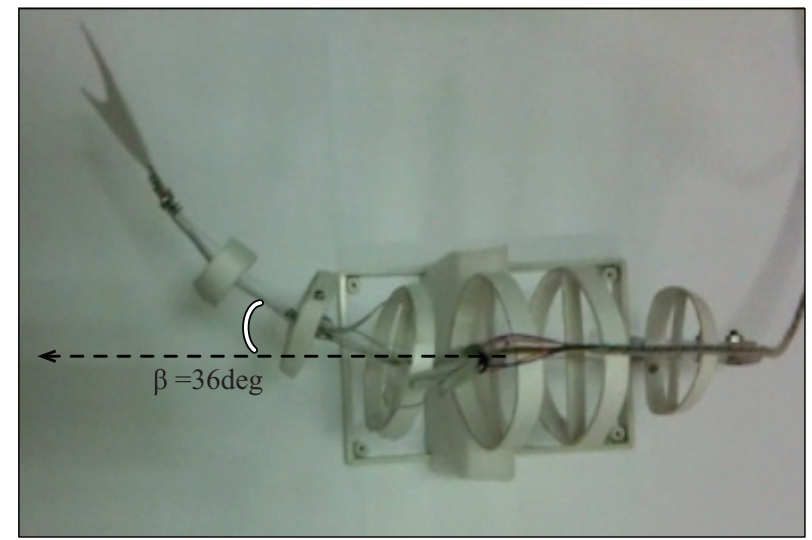

Figure 13. Bending under SMA overloading [59].

\subsubsection{Control architecture}

A key feature of SMAs is the possibility to develop closed loop control systems without the need of external sensor hardware. The feed back signal is provided by the detection of inner electrical resistance, that allows an indirect measurement of the temperature.

The main components are described in the following. A micro controller implements the PID algorithm. The PID controller receives the input reference position (set point) and the feedback of SMA's voltage and current that allows calculating the heating current to drive the SMA actuator. The digital output of the PID controller is converted to a reference current in 
two steps. First, it is converted into an analog signal using a 2-wire serial 8-Bit DAC (Digital to Analog Converter) with Rail-to-Rail outputs. Then, a Voltage Controlled Current Source (VCCS) transforms the DC voltage in a constant current that feeds the SMA. This stage has a power consumption of less than $10 m A$.

The DAC output ranges from 0 to 5 Volts with a resolution of $0.02 \mathrm{~V}$. The measured voltage (VSMA) and current (ISMA) on the SMA fiber are fed-back to the micro controller in order to close the control loop. The hardware used (16F690-PIC) had a 12-bits A/D converter with a resolution of $0.537 \mathrm{mV}$ (considering the maximum voltage measured at the SMA VSMA = $0.55 \mathrm{~V}$.

On the other hand, taking into consideration the maximum current through the wire $(500 \mathrm{~mA})$, SMA resistance variations about $1.074 \mathrm{~m} \Omega$ can be measured. Therefore, since the maximum variation in the SMA length is $0.34 \mathrm{~cm}$, and the maximum variation of the resistance is $1.6 \Omega$, the theoretical position error of the system based on the SMA length is $0.067 \%$. i.e., $0.12 \mathrm{~mm}$.

\subsection{BaTboT: a biologically-inspired bat-like aerial robot}

BaTboT is a bio-inspired bat robot that uses Shape Memory Alloys (SMAs) as artificial muscles for powering the morphing motion of the wings. The morphing motion is related to the capacity of the robot to modulate its wings by contracting and extending the membrane in sync with the flapping motion. It is precisely this characteristic what makes biological bats more agile to maneuver than any other flying creature within the same Reynolds number range $\left(10^{3}-10^{4}\right)$ [66], [22]. In addition, biological studies in [32], [58] have revealed that real bats are able to maneuver because of the inertial changes produced by the wings' modulations. Attempting to mimic this functionality using an artificial counterpart -BaTboTmainly presents a twofold challenge: i) biomechanical design of the wings, and ii) proper control/actuation to module BaTboT's wings.

Prior work in [5] presented experimental results regarding both challenges. The investigation carried out in [5] does not only describes the biomechanical design of BaTboT's wings, but also focuses on evaluating the implications of using SMAs as artificial muscles to power the change of wing's morphology. Figure 14 shows the design-flow process to evaluate key issues of SMA performance and their implications to the application at hand.

The use of SMAs as artificial muscles has been concretely evaluated in terms of two issues:

- Functionality: SMA Power-to-Force.

- SMA Performance: actuation speed and fatigue.

\subsubsection{Functionality}

The SMA actuators shown in Fig. 14 (step-1) are the commercial Migamotor NanoMuscle model RS-70-CE [49]. Each NanoMuscle consists of several short strips of SMA NiTi wire with a thickness of $150 \mu \mathrm{m}$ attached to opposite ends of six metal strips stacked in parallel. Each SMA segment pulls the next strip about $0.67 \mathrm{~mm}$ relative to the previous strip, and the relative movements sum to make a stroke of $4 \mathrm{~mm}$. As depicted, two Migamotors muscles have been arranged into an antagonistic configuration working as artificial biceps and triceps that provide the rotation motion of the wing elbow's joint $\theta_{3}$. The range of motion of the joint 


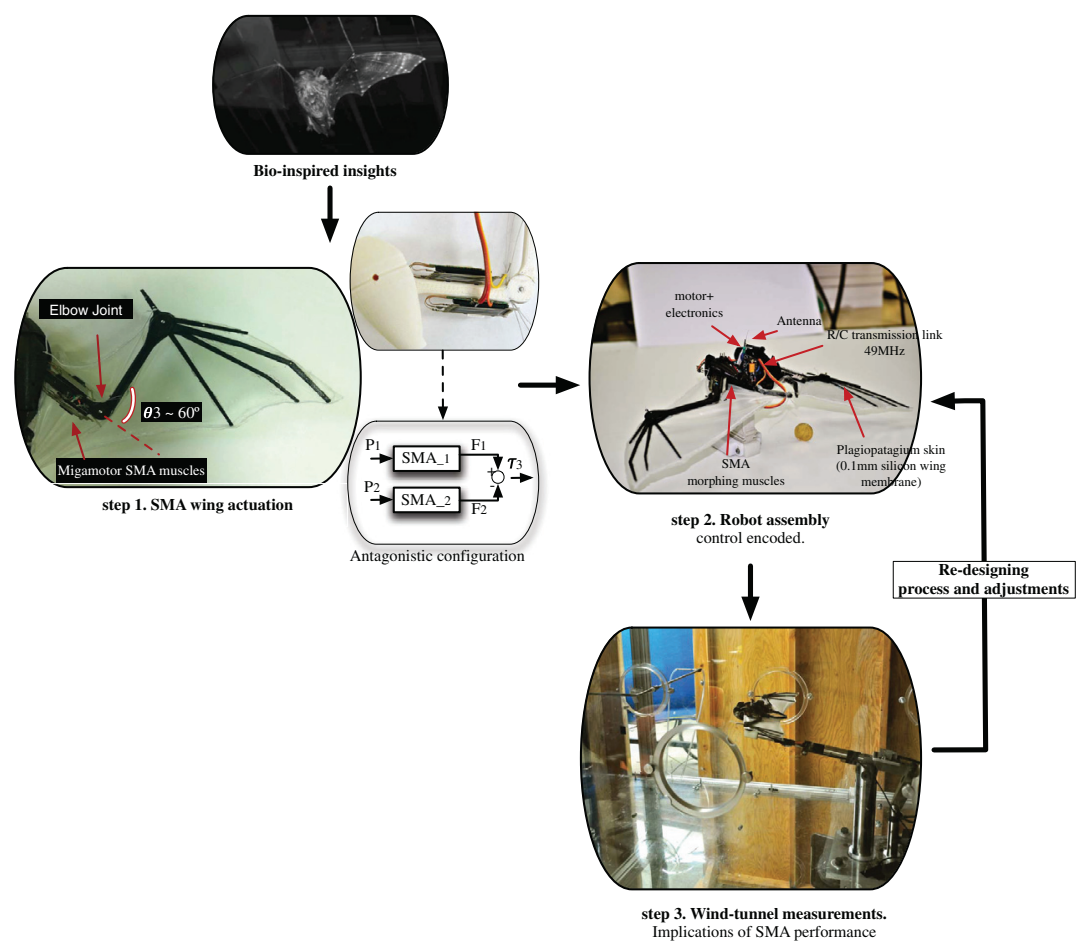

Figure 14. Flow-process for SMA evaluation in the BaTboT prototype [5].

is about $60^{\circ}$. The joint motion is achieved when each SMA actuator contracts upon heating, thus generating a pull-force $\left(F_{s m a}\right)$. Because both actuators are connected to the joint in an antagonistic fashion, the pull-force $F_{s m a}$ generates a joint torque, denoted as $\tau_{3}$. Therefore, each actuator requires an input heating power $P$, to produce and output torque $\tau_{3}$. The input heating power $(P)$ is proportional to the input electrical current $(I): P=I^{2} R$, being $R$ the nominal electrical resistance of the NiTi wires, $R=8.5 \Omega$.

In [5] simulations and experiments have been carried out aimed at quantifying the Power-to-Force tradeoff of the SMA muscles working under two operation modes: nominal and overloaded. To the application at hand, nominal-mode implies an input heating current ( $I$ ) between $175 \mathrm{~mA}$ and $350 \mathrm{~mA}$, whereas overloaded-mode, between $\sim 400 \mathrm{~mA}$ and $600 \mathrm{~m} A$. Overloading allows for increasing the heating-time of the SMA, therefore increasing the contraction speed and the overall actuation frequency. However, overloading must be monitored to avoid overheating issues that may cause physical damage of the shape memory effect. In [5], SMA limitations were found under simulation and validated under experimentation.

Fig.15 shows the Power-to-Force tradeoffs: i) simulation using a SMA phenomenological model, ii) SMA response using an identified model, and iii) experimental measurements of output torque. Herein, our goal is not focused on describing the models, but on comparing 
the correlations between models (i)-(ii) and experimental data (iii). Further details about the models can be found in [5].
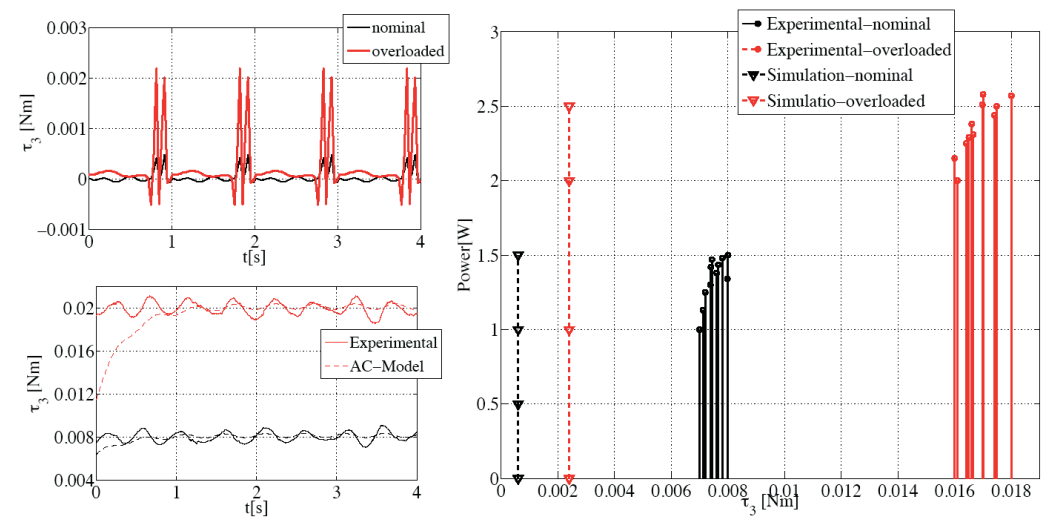

Figure 15. SMA Power-to-Force response. The input power is of the form: $a+b \sin (2 \pi f t)$. a) Simulation of elbow's torque response under nominal and overloaded SMA operation, b) AC small-signal response of SMAs at $\mathrm{f}=2 \mathrm{~Hz}, \mathrm{c}$ ) maximum peak-values of output torque as a function of the input power

In order to simulate how the SMA muscles response upon electrical heating, a phenomenological model was used. This model is composed by thermo-mechanical equations that describe how the temperature, strain, and stress of the SMA change during its hysteresis loop, going from austenite to martensite and vice versa. Likewise, the SMA phenomenological actuation model is coupled with the dynamics equations that govern the motion of the robot's wing (refer to [5] for further details).

Figure 15a shows simulation results of joint torques achieved by applying nominal and overloaded values of input power of the form: $a+b \sin (2 \pi f t)$, where $a$ is the mean input power $P$, the term $b$ is the small-magnitude of the sinusoidal component, and $f$ is the commanded frequency for SMA contraction. Simulation data determines that by applying a nominal input power of $P=1.04 \mathrm{~W}$, the SMAs are able to generate a torque around the elbow joint of $\tau_{3}=0.0007 \mathrm{Nm}$, whereas by applying an overloaded input power of $P=2.57 \mathrm{~W}$, the output torque can be increased up to $\tau_{3}=0.0022 \mathrm{Nm}$. This implies that by increasing the input power about 2.5 times, the output torque can be increased about three times.

In order to verify the accuracy of simulation results, Fig.15c shows experimental measurements of output torques. In this trial, several profiles of input power were applied, and the respective forces were measured. Note that under nominal-mode, the output torque varies between $0.007-0.008 \mathrm{Nm}$, whereas under overloading-mode, from $0.016-0.018 \mathrm{Nm}$. Likewise, at the left side of the plot, the maximum peaks of nominal and overloaded torques corresponding to the simulation in Fig. 15 a, are also shown. One can note there is almost an order of magnitude of difference between simulation and experimental data. The error is produced because the phenomenological model does not take into account the joint friction, and even most important, the anisotropic loading of the silicone-based membrane. However, despite the error in magnitude, note that by doubling the input heating power, the measured 


\begin{tabular}{|c|c|c|c|c|c|}
\hline & Parameter & Theoretical $^{1}$ & Simulation $^{2}$ & Experimental $^{3}$ & AC-model $^{4}$ \\
\hline \multirow{5}{*}{ Nominal } & Input power P [W] & 0.26 & 1.04 & $0.87-1.5$ & 1.36 \\
& Input current I [mA] & 175 & 350 & $320-420$ & 400 \\
& SMA Pull-force F [N] & 0.012 & 0.007 & $0.07-0.08$ & 0.08 \\
& Output torque $\operatorname{tau}_{3}[\mathrm{Nm}]$ & 0.0012 & 0.0007 & $0.007-0.008$ & 0.008 \\
\hline \multirow{5}{*}{ Overloaded } & Input power P [W] & - & 2.57 & $2-2.57$ & 3.06 \\
& Input current I [ $m A]$ & - & 550 & $485-550$ & 600 \\
& SMA Pull-force $F[N]$ & - & 0.022 & $0.16-0.18$ & 0.2 \\
& Output torque tau $_{3}[N m]$ & - & 0.0022 & $0.016-0.018$ & 0.02 \\
\hline
\end{tabular}

Table 2. SMA-muscle Power-to-Force performance. ${ }^{1}$ Nominal values can be found in Migamotors website [49]. The manufacturer does not provide overloading values. ${ }^{2}$ Simulation data corresponds to Fig. 15 a. Please refer to Fig.9 in [5] for details about the SMA phenomenological model. ${ }^{3}$ Experimental data corresponds to Fig. 15 c. ${ }^{3}$ Identified AC Power-to-force model response. Data corresponding to Fig. $15 \mathrm{~b}$.

output torque increases about 2.5 times. This scaling factor is quite similar compared to the simulation prediction.

The use of a phenomenological SMA model can provide a useful insight into the implications of overheating, by increasing the input power beyond the limits of SMA stress, and thus defining the upper threshold for overloading when using the real system. Unfortunately, the phenomenological model has not proven to be accurate for modeling the response of the SMAs when applying small-signal power inputs. Therefore, the next step is to identify the Power-to-Force AC behavior of the SMA actuators.

As observed by [11], [71] the AC response of NiTi SMA wires behaves as a first order low-pass filter. In [40], [19], [20] similar first-order behavior in their SMA actuator response had also been observed. Therefore in [5], we have followed a similar frequency response analysis procedure for finding a transfer function model that matches the measured gain and phase data of the frequency range of interest. Fig. 16 shows the results.

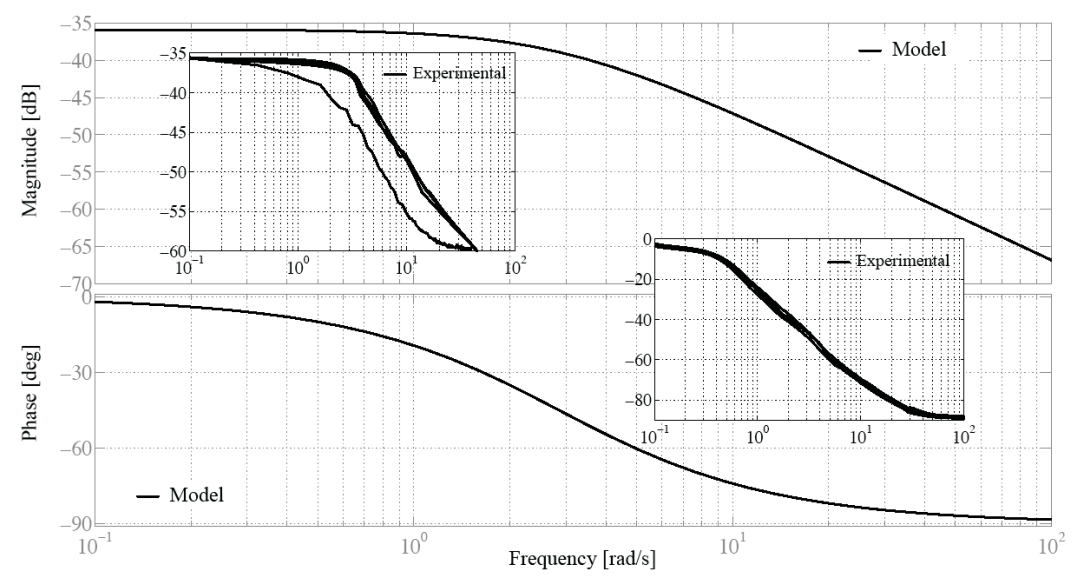

Figure 16. (Experiment VS model) Bode magnitude and phase plots for NiTi 150 $\mu \mathrm{m}$ SMA Migamotor actuator. Input heating power of the form: $a+b \sin (2 \pi f t)$. 
The suitable transfer function that fits the experimental data from Fig. 16 is: $\tau_{3}(s)=$ $0.016(0.35 s+1)^{-1} P(s)$. The response of the AC model is shown in Fig. $15 \mathrm{~b}$. The AC model is compared against the experimental response of the SMA actuator to the AC small-signal. Note that by applying an input power of $\sim 1.36 \mathrm{~W}(\mathrm{I}=400 \mathrm{~mA})$, the output torque $\tau_{3}$ stabilizes around $\sim 0.008 \mathrm{Nm}$. This response corresponds to the real nominal mode of the SMA actuator. Increasing the input power up to $\sim 3.06 \mathrm{~W}$ (overloaded mode, $I=600 \mathrm{~mA}$ ), the registered output torque stabilizes around $\sim 0.02 \mathrm{Nm}$. In both experiments the anisotropic loading of the $0.1 \mathrm{~mm}$ silicone-based membrane has been taken into account.

Note how well the AC model correlates against the real behavior of the SMA muscles. A detailed comparison of the obtained data is summarized in Table 2. In conclusion, to properly evaluate the functionality of using SMA muscles based on the criteria of the application at hand:

- First of all, identify the required force the SMA muscle must provide to drive the motion of the robot. This quantification requires a dynamic model of the robot.

- Use a phenomenological model for defining the limits of SMA actuation. The best models in the literature can be found in [69],[1],[7],[8],[10].

- Classify the response of the SMA in terms of nominal and overloaded input power. Verify that under overloaded operation, SMAs are safe from overheating, and yet achieving the desired output torque - to - input power tradeoff.

- Using the real system, apply an AC input power of the form $a+b \sin (2 \pi f t)$, being $a$ the mean power (nominal or overloaded), $b$ the small-signal amplitude of the sinusoidal component, and $f$ the desired actuation frequency. Subsequently, measure the small changes of pulling-force generated by the SMA when contracting. Note that forces can be mapped to torques (if using a rotational joint).

- Follow the frequency response analysis procedure described in [11], [71]. Note the AC behavior of a NiTi SMA wire resemble a first order low-pass filter. Find the transfer function that matches the data. The Power-to-Force model of the SMA will be of the form: $F(s)=k(\tau s+1)^{-1} P(s)$. Use the identified model for control tuning. Note that a simple PID control technique is suitable for driving SMA actuation. Depending on the available sensors, force control or position control could be applied. The following section provides a brief insight about the advantages and implications of using both control schemes: force or position feedback. For detailed insights about SMA force control, refer to [71], whereas for SMA position control, refer to [5], and [59].

\subsubsection{SMA Performance: actuation speed and fatigue}

As concluded in [29], [57], [11], [17], [76], PID technique is sufficient for allowing accurate and faster SMA actuation. The key issue for achieving an outstanding SMA performance does not necessarily depend on the control technique, but in complementary mechanisms that monitor the lower and upper limits of input power.

In [11], and [71], these mechanisms are introduced: i) anti-slack, and ii) anti-overload. The former deals with the two-way shape memory effect [39], improving accuracy and speed, 
whereas the latter limits the amount of input heating power to prevent physical damage when SMAs are overloaded. As a result, the force controller presented in [71] was capable of tracking fast and accurate force references when compared with other works reported in [20], [84].

The improvement in accuracy and speed are due to two factors: i) by avoiding wire entanglement, and ii) by ensuring the passive wire of the antagonistic configuration does not cool completely. Wire entanglement can be produced when the SMA wires extended upon cooling. The passive SMA wire can develop a few millimeters of slack as it cools, which consequently affects the accuracy of the control. This slack-phenomenon is only presented in the antagonistic configuration, due to the two-way shape memory effect produced by each actuator.

To avoid the aforementioned issues, the anti-slack mechanism defines a minimum threshold of input heating power that ensures the inactive wire does not cool completely. The improvement in actuation speed is due to the fact that the already-warm SMA wire can begin to pull as soon as the heating current is raised, whereas a cold wire would first need to be raised to its operating temperature. It has been observed from experimental results in [71] and [5] that a suitable minimum threshold of input heating power is about $10 \%$ of the power applied.

On the other hand, the anti-overload mechanism is in charge of ensuring that the maximum input power does not increase above an upper limit. This approach avoids overheating the SMAs in case the controller delivers a large amount of power to the wires. As mentioned before, this upper limit can be found using a phenomenological model, or by performing real measurements of SMA temperature and stress on the wires.

Both anti-slack and anti-overload mechanisms are key for improving on SMA performance under a force control architecture. The advantages of using a force-control scheme are twofold: i) high-bandwidth response, and ii) SMA fatigue avoidance. High-bandwidth response requires the use of force sensors capable of providing the force feedback. It has also been demonstrated in [71], that by using high-bandwidth force feedback, limit cycles of SMA operation are eliminated.

Nevertheless, for some systems, the use of force sensors could be a hardware limitation. In [5], it has been demonstrated that both anti-slack and anti-overload mechanisms can be implemented in a position control scheme. The position feedback can be achieved by measuring the electrical resistance of the SMA wires, which is a linear function of the strain. The key disadvantage of using a position scheme that forces the SMA to behave in overloaded operation mode relies on fatigue. As experimentally observed in [5], overloaded operation mode could be maintained only for about five minutes of SMA continuous operation before decreasing performance to nominal mode. For the application at hand, overloaded mode implied an actuation frequency of $2.5 \mathrm{~Hz}$, while nominal mode, an actuation frequency of $1.3 \mathrm{~Hz}$. In this case fatigue issues caused a decrease in actuation speed performance about $56 \%$. Further investigations shall be devoted to quantifying the lifetime of SMAs when subjected to higher stresses and larger heating currents within a position control scheme. 


\section{Conclusions}

SMA technology allows the development of a wide variety of robotics designs, exploiting their advantages in terms of weight, volume and sensing capabilities. Their particular features open a wide range of possibilities hardly achievable with classical technology: hydrofoils, morphing shapes, and hydrostats are just some of the new concepts that have been made possible to develop thanks to SMAs. The prototypes described in this chapter have been classified first according to the operation environment (water, air and ground) and second according to their application in the robotics system.

Researchers have successfully overcome SMA disadvantages in terms of power consumption, actuation speed and low strain, by:

- Finding suitable niches of application

- Designing dedicated mechatronics

- Developing ad-hoc control strategies

Biomimetics has emerged as a very promising field together with bio-medical applications. Even if SMAs cannot substitute classical servomotor and hydraulic technology in general, niches can be found where they can effectively compete with, and even outperform standard actuation technology. Clearly, merely replacing servomotors with SMA-based actuators in classical linear-actuation mechanic setups would make little sense. But dedicated mechatronic design, such as embedding SMA fibers in silicone obtaining morphing materials, bending of continuous structures and hydrostats make the best out of this technology, regardless the relatively small strain achievable from the fibers. Finally, cleverly designed control strategies, that exploit the knowledge of the physics of the material and of its behavior over time, coupled with dedicated mechanic setups can dramatically reduce response time.

Our experience with SMA-based actuators has revealed a relatively little considered drawback, which relates to fatigue. In fact, extensive testing with our prototypes has demonstrated that even if SMA performance can be improved by overloading, the effects of overloading disappear quite rapidly. We believe that further investigations shall be devoted to study the behavior of the alloys when exposed to high stresses and large heating currents over a large period of time.

In conclusion, we can say that Shape Memory Alloys have demonstrated great capabilities, as it has been demonstrated by this review of the state of the art. Most importantly, they have the potential to be used in future robotic systems: the more researchers will apply and study them, the more their limitations are smoothed and their employment becomes more effective. After all, they are being extensively investigated only since a short time in comparison with electric motors and hydraulic actuators, that can boast more than a century of history.

\section{Author details}

William Coral, Claudio Rossi, Julian Colorado, Daniel Lemus and Antonio Barrientos

Centre for Automation and Robotics (CAR) of the Technical University of Madrid, Spain

CSIC (National Council for Scientific research) 


\section{Acknowledgements}

This work was supported by the Robotics and Cybernetics Group at the Centre for Automation and Robotics (CAR) UPM-CSIC, and funded under the project ROBOCITY 2030 sponsored by the Community of Madrid (S-0505/DPI/000235) and The project ROTOS (MultiRobot Systems for Protection of large outdoor infrastructures) sponsored by the Ministry of Science And Innovation of Spain (DPI2010-17998)

\section{References}

[1] Brinson, L. C. [1993]. One-Dimensional Constitutive Behavior of Shape Memory Alloys: Thermomechanical Derivation with Non-Constant Material Functions and Redefined Martensite Internal Variable, Journal of Intelligent Material Systems and Structures 4(2): 229-242.

URL: $h$ ttp://jim.sagepub.com/cgi/doi/10.1177/1045389X9300400213

[2] Bunget, G. \& Seelecke, S. [2010]. BATMAV: a 2-DOF bio-inspired flapping flight platform, Proceedings of SPIE 7643: 76433B.

[3] Caldwell, D. G. \& Taylor., P. [1988]. Artificial muscles as robotic actuators, IFAC Robot Control, Karlsruhe, pp. 401-406.

[4] Case Western Reserve University. Continuous Wave Peristaltic Locomotion, In: Center For Biologically Inspired Robotics Research, 2012 [2012].

URL: http://biorobots.cwru.edu/projects/softworm/

[5] Colorado, J., Barrientos, A., Rossi, C. \& Breuer, K. [2012]. Biomechanics of smart wings in a bat robot: morphing-wings using SMA actuators, Bioinspiration \& Biomimetics 7(3).

URL: $h$ ttp://iopscience.iop.org/1748-3190/7/3/036006

[6] Dynalloy [2012, Available from: http://www.dynalloy.com/TechDataWire.php]. Dynalloy, Inc. FLEXINOL®Actuator Wire In: Technical and Design Data.

URL: http://www.dynalloy.com/TechDataWire.php

[7] Elahinia, M. H. [2004]. Effect of System Dynamics on Shape Memory Alloy Behavior and Control, PhD thesis, Virginia Polytechnic Institute and State University.

[8] Elahinia, M. H. \& Ahmadian, M. [2005]. An enhanced SMA phenomenological model: I. The shortcomings of the existing models, Smart Materials and Structures 14(6): 1297-1308. URL: http://stacks.iop.org/0964-1726/14/i=6/a=022? key=crossref.356645df188b7494fe51e5de 94b4f281

[9] Eren, Y., Mavroidis, C. \& Nikitczuk, J. [2002]. B-Spline Based Adaptive Control of Shape Memory Alloy Actuated Robotic Systems, Dynamic Systems and Control, Vol. 2002, ASME, pp. $471-478$.

URL: http://dx.doi.org/10.1115/IMECE2002-33425

[10] Esfahani, E. T. \& Elahinia, M. H. [2007]. Stable Walking Pattern for an SMA-Actuated Biped, 12(5): 534-541.

[11] Featherstone, R. [2008]. An Architecture for Fast and Accurate Control of Shape Memory Alloy Actuators, The International Journal of Robotics Research 27(5): 595-611.

URL: http://ijr.sagepub.com/cgi/doi/10.1177/0278364908090951 
[12] Featherstone, R. \& Teh, Y. H. [2004]. Improving the Speed of Shape Memory Alloy Actuators by Faster Electrical Heating, 9th International Symposium on Experimental Robotics pp. 1-10.

[13] Fujita, H. [1989]. Studies of micro actuators in Japan, International Conference on Robotics and Automation, IEEE Comput. Soc. Press, pp. 1559-1564.

URL: $h$ ttp://ieeexplore.ieee.org/lpdocs/epic03/wrapper.htm?arnumber $=100200$

[14] Fukuda, T. \& Asaka, K. [2003]. A new type of fish-like underwater microrobot, IEEE/ASME Transactions on Mechatronics 8(1): 136-141.

URL: http://ieeexplore.ieee.org/lpdocs/epic03/wrapper.htm?arnumber $=1187383$

[15] G. K. Batchelor, U. o. C. [2000]. An Introduction to Fluid Dynamics, cambridge edn, Cambridge University Press, Cambridge.

URL: http://www.cambridge.org/gb/knowledge/isbn/item1161783/?site_locale=en_GB

[16] Rediniotis, O. K., Wilson, L. N., Lagoudas, D. C. \& Dimitris, C. [2002]. Development of a Shape-Memory-Alloy Actuated Biomimetic Hydrofoil, Intelligent Materials Systems and Structures 13(1): 35-49.

[17] Gorbet, R. B. \& Russell, R. A. [1995]. A novel differential shape memory alloy actuator for position control, Robotica 13(4): 423-430.

URL: http://www.journals.cambridge.org/abstract_S0263574700018853

[18] Granito, M. [2011]. S.M.A. Shape Memory Alloy Cooling System by Peltier Cells: A Cooling System for Shape Memory Alloy Based on the Use of Peltier Cells, LAP Lambert Acad. Publ. URL: http://books.google.es/books? id=IFvSygAACAAJ

[19] Grant, D. [1999]. Accurate and rapid control of shape memory alloy actuators, PhD thesis.

[20] Grant, D. \& Hayward, V. [1997]. Variable structure control of shape memory alloy actuators, Control Systems Magazine IEEE 17(3): 80-88.

URL: $h$ ttp://ieeexplore.ieee.org/xpls/abs_all.jsp?arnumber $=588180$

[21] Hashimoto, M., Tabata, T. \& Yuki, T. [1999]. Development of electrically heated SMA active forceps for laparoscopic surgery, IEEE International Conference on Robotics and Automation Cat No99CH36288C 3(May): 2372-2377.

URL: http://ieeexplore.ieee.org/lpdocs/epic03/wrapper.htm?arnumber $=770460$

[22] Hedenström, A., Johansson, L. C. \& Spedding, G. R. [2009]. Bird or bat: comparing airframe design and flight performance., Bioinspiration biomimetics 4(1): 015001.

URL: http://www.ncbi.nlm.nih.gov/pubmed/19258691

[23] Ho, M., McMillan, A. B., Simard, J. M., Gullapalli, R. \& Desai, J. P. [2011]. Toward a Meso-Scale SMA-Actuated MRI-Compatible Neurosurgical Robot: Robotics, IEEE Transactions on, Robotics IEEE Transactions on PP(99): 1-10.

[24] Homsy, G., Aref, H., Breuer, K.S., Hochgreb, S., Powell, K., Munson, B. \& Robertson, C. [2008]. Multimedia Fluid Mechanics DVD-ROM, second edi edn, Cambridge University Press.

[25] Honma, D., Miwa, Y. \& Iguchi. [1985]. Micro Robots and Micro Mechanisms Using Shape Memory Alloy to Robotic Actuators, Robotic Systems 2(1): 3-25.

[26] Hunter, I. W. \& Lafontaine, S. [1992]. A comparison of muscle with artificial actuators, SolidState Sensor and Actuator Workshop 5th Technical Digest IEEE, pp. 178-185.

URL: http://ieeexplore.ieee.org/xpls/abs_all.jsp?arnumber $=228297$ 
[27] Hunter, I. W., Lafontaine, S., Hollerbach, J. M. \& Hunter, P. J. [1991]. Fast reversible NiTi fibers for use in microrobotics, Micro Electro Mechanical Systems, Ieee, pp. 166-170.

URL: http://ieeexplore.ieee.org/lpdocs/epic03/wrapper.htm?arnumber $=114789$

[28] Ihálcz, I. M. [2001]. Fundamental characteristics and design method for nickel-titanium shape memory alloy, Periodica Polytechnica Mechanical Engineering 45(1): 75-86.

URL: http://www.pp.bme.hu/me/2001_1/pdf/me2001_1_10.pdf

[29] Ikuta, K. [1990]. Micro/miniature shape memory alloy actuator, International Conference on Robotics and Automation, Vol. 3, IEEE Comput. Soc. Press, pp. 2156-2161.

[30] Ikuta, K., Tsukamoto, M. \& Hirose, S. [1988]. Shape memory alloy servo actuator system with electric resistance feedback and application for active endoscope, International Conference on Robotics and Automation, Vol. 1, IEEE, pp. 427-430.

URL: http://ieeexplore.ieee.org/xpls/abs_all.jsp?arnumber $=12085$

[31] Ikuta, K., Tsukamoto, M. \& Hirose, S. [1991]. Mathematical model and experimental verification of shape memory alloy for designing micro actuator, IEEE Micro Electro Mechanical Systems, pp. 103-108.

[32] Iriarte-Díaz, J., Riskin, D. K., Willis, D. J., Breuer, K. S. \& Swartz, S. M. [2011]. Whole-body kinematics of a fruit bat reveal the influence of wing inertia on body accelerations., Journal of Experimental Biology 214(Pt 9): 1546-1553.

URL: http://www.ncbi.nlm.nih.gov/pubmed/21490262

[33] Kim, B., Kim, D.-h., Jung, J. \& Park, J.-o. [2005]. A biomimetic undulatory tadpole robot using ionic polymer-metal composite actuators, Smart Materials and Structures 14(6): 1579-1585.

URL: http://stacks.iop.org/0964-1726/14/i=6/a=051? key=crossref.f252f55e78ad58af50f72847f0 cd41a3

[34] Kim, B., Lee, M. G., Lee, Y. P., Kim, Y. \& Lee, G. [2006]. An earthworm-like micro robot using shape memory alloy actuator, Sensors and Actuators A: Physical 125(2): 429-437.

URL: http://linkinghub.elsevier.com/retrieve/pii/S0924424705003067

[35] Kim, B., Park, S. \& Park, J.-O. [2009]. Microrobots for a capsule endoscope, 2009 IEEE/ASME International Conference on Advanced Intelligent Mechatronics pp. 729-734.

URL: http://ieeexplore.ieee.org/lpdocs/epic03/wrapper.htm?arnumber $=5229926$

[36] Kim, S., Hawkes, E., Choy, K. \& Joldaz, M. [2009]. Micro artificial muscle fiber using NiTi spring for soft robotics, Robots and Systems pp. 2228-2234.

URL: http://ieeexplore.ieee.org/xpls/abs_all.jsp?arnumber $=5354178$

[37] Koh, J. [2009]. Omegabot: Biomimetic inchworm robot using sma coil actuator and smart composite microstructures (scm), Robotics and Biomimetics (ROBIO), 2009 pp. 1154-1159.

URL: http://ieeexplore.ieee.org/lpdocs/epic03/wrapper.htm?arnumber $=5420752$ http:// ieeexplore. ieee.org/xpls/abs_all.jsp?arnumber $=5420752$

[38] Koh, J.-s. \& Cho, K.-j. [2010]. Omegabot: Crawling robot inspired by Ascotis Selenaria, International Conference on Robotics and Automation, IEEE, pp. 109-114.

URL: $h$ ttp://ieeexplore.ieee.org/lpdocs/epic03/wrapper.htm?arnumber $=5509425$

[39] Kohl, M. [2004]. Shape Memory Microactuators, Springer.

URL: http://www.amazon.com/Shape-Memory-Microactuators-Manfred-Kohl/dp/3540206353

[40] Kuribayashi, K. [1986]. A New Actuator of a Joint Mechanism Using TiNi Alloy Wire, The International Journal of Robotics Research 4(4): 47-58. 
URL: http://ijr.sagepub.com/cgi/doi/10.1177/027836498600400404

http://ijr.sagepub.com/cgi/content/abstract/4/4/47

[41] Kuribayashi, K. [1991]. Improvement of the Response of an SMA Actuator Using a Temperature Sensor, The International Journal of Robotics Research 10(1): 13-20.

URL: http://ijr.sagepub.com/cgi/doi/10.1177/027836499101000102

[42] Cecilia Laschi, Matteo Cianchetti, Barbara Mazzolai, Laura Margheri, Maurizio Follador \& Paolo Dario [2012]. Soft Robot Arm Inspired by the Octopus, Advanced Robotics 26: 709-727.

[43] Li, W. D., Guo, W., Li, M. T. \& Zhu, Y. H. [2006]. Design and Test of a Capsule Type Endoscope Robot with Novel Locomation Principle, 2006 9th International Conference on Control Automation Robotics and Vision 20(02): 1-6.

URL: http://ieeexplore.ieee.org/lpdocs/epic03/wrapper.htm?arnumber $=4150137$

[44] Liu, S.-H. \& Yen, J.-Y. [2008]. Sensor fusion in a SMA-based hexapod bio-mimetic robot, Workshop on Advanced robotics and Its Social Impacts, IEEE, pp. 1-6.

URL: http://ieeexplore.ieee.org/lpdocs/epic03/wrapper.htm? arnumber $=4653611$

[45] Liu, X., Luo, H.-Y., Liu, S.-P. \& Wang, D.-F. [2011]. Pilot study of SMA-based expansion device for transanal endoscopic microsurgery, 2011 International Conference on Machine Learning and Cybernetics, IEEE, pp. 1420-1424.

URL: $h$ ttp://ieeexplore.ieee.org/lpdocs/epic03/wrapper.htm?arnumber $=6016875$

[46] M. Hashimoto, Takeda, M., Sagawa, H., Chiba, I. \& Sato, K. [1985]. Application of shape memory alloy to robotic actuators, Journal of Robotic Systems 2(1): 3-25.

[47] McNaney, J. [2003]. An experimental study of the superelastic effect in a shape-memory Nitinol alloy under biaxial loading, Mechanics of Materials 35(10): 969-986.

URL: http://linkinghub.elsevier.com/retrieve/pii/S0167663602003101

[48] Menciassi, A., Gorini, S., Pernorio, G. \& Dario, P. [2004]. A SMA Actuated Artificial Earthworm, IEEE International Conference on Robotics and Automation 2004 Proceedings ICRA 042004 4: 3282-3287 Vol.4.

URL: $h$ ttp://ieeexplore.ieee.org/lpdocs/epic03/wrapper.htm?arnumber $=1308760$

[49] Migamotors [n.d.]. Migamotors Company, 2012, Avaliable from: http://www. migamotors.com/. URL: http://www.migamotors.com/

[50] M.W.M. van der Wijst [1998]. Shape control of structures and materials with shape memory alloys, PhD thesis, Universiteit Eindhoven.

URL: http://en.scientificcommons.org/17599133

[51] Nguyen, Q., Park, H., Byun, D. \& Goo, N. [2010]. Recent progress in developing a beetle-mimicking flapping-wing system, World Automation Congress (WAC), pp. 1-6.

[52] Pons, J. L. [2005]. Emerging Actuator Technologies: A Micromechatronic Approach, Wiley.

[53] Pornsin-sirirak, T., Tai, Y., Nassef, H. \& Ho, C. [2001]. Titanium-alloy MEMS wing technology for a micro aerial vehicle application, Sensors and Actuators A: Physical 89(1-2): 95-103.

URL: http://linkinghub.elsevier.com/retrieve/pii/S0924424700005276

[54] Punning, A., Anton, M., Kruusmaa, M. \& Aabloo, A. [2004]. A Biologically Inspired Ray-like Underwater Robot with Electroactive Polymer Pectoral Fins, IEEE Confrence "Mechatronics and Robotics. 
[55] Rabinovich, M., Varona, P., Selverston, A. \& Abarbanel, H. [2006]. Dynamical principles in neuroscience, Reviews of Modern Physics 78(4): 1213-1265.

URL: http://link.aps.org/doi/10.1103/RevModPhys.78.1213

[56] Raynaerts, D. \& Brussel, H. V. [1991]. Development of a SMA high performance robotic actuator, Fifth International Conference on Advanced Robotics Robots in Unstructured Environments, Ieee, pp. 61-66.

URL: http://ieeexplore.ieee.org/lpdocs/epic03/wrapper.htm?arnumber $=240475$

[57] Reynaerts, D. \& Brussel, H. V. [1998]. Design aspects of shape memory actuators, Mechatronics 8(6): 635-656.

URL: http://linkinghub.elsevier.com/retrieve/pii/S0957415898000233

[58] Riskin, D. K., Iriarte-Díaz, J., Middleton, K. M., Breuer, K. S. \& Swartz, S. M. [2010]. The effect of body size on the wing movements of pteropodid bats, with insights into thrust and lift production., Journal of Experimental Biology 213(Pt 23): 4110-4122.

URL: http://www.ncbi.nlm.nih.gov/pubmed/21075953

[59] Rossi, C., Colorado, J., Coral, W. \& Barrientos, A. [2011]. Bending continuous structures with SMAs : a novel robotic fish design, Bioinspiration biomimetics 045005(4): 045005.

URL: $h$ ttp://iopscience.iop.org/1748-3190/6/4/045005

[60] Rossi, C., Coral, W. \& Barrientos., A. [2010]. SMA Control for Bio-mimetic Fish Locomotion, International Conference on Informatics in Control, Automation and Robotics (ICINCO), Madeira.

[61] Seok, S., Onal, C. D., Wood, R., Rus, D. \& Kim, S. [2010]. Peristaltic locomotion with antagonistic actuators in soft robotics, 2010 IEEE International Conference on Robotics and Automation 0060: 1228-1233.

URL: http://ieeexplore.ieee.org/lpdocs/epic03/wrapper.htm?arnumber $=5509542$

[62] Shi, Z., Liu, D., Ma, C. \& Zhao, D. [2011]. Accurate controlled Shape Memory Alloy Actuator for Minimally Invasive Surgery, Self pp. 817-822.

[63] Shin, B., Kim, H.-Y. \& Cho, K.-J. [2008]. Towards a biologically inspired small-scale water jumping robot, 2008 2nd IEEE RAS \& EMBS International Conference on Biomedical Robotics and Biomechatronics, IEEE, pp. 127-131.

URL: $h$ ttp://ieeexplore.ieee.org/lpdocs/epic03/wrapper.htm? arnumber $=4762896$

[64] Shin, D. D., Mohanchandra, K. P. \& Carman, G. P. [2004]. High frequency actuation of thin film NiTi, Sensors And Actuators 111(2-3): 166-171.

URL: http://linkinghub.elsevier.com/retrieve/pii/S0924424703004771

[65] Suleman, A. \& Crawford, C. [2008]. Design and testing of a biomimetic tuna using shape memory alloy induced propulsion, Computers and Structures (86): 491?499.

[66] Swartz, S. M., Bishop, K. L. \& Ismael-Aguirre, M. F. [2005]. Dynamic complexity of wing form in bats: implications for flight performance, Functional and evolutionary ecology of bats pp. 110-130.

[67] Tadesse, Y., Thayer, N. \& Priya, S. [2009]. Tailoring the Response Time of Shape Memory Alloy Wires through Active Cooling and Pre-stress, Journal of Intelligent Material Systems and Structures 21(1): 19-40.

URL: http://jim.sagepub.com/cgi/doi/10.1177/1045389X09352814

[68] Tadesse, Y., Villanueva, A., Haines, C., Novitski, D., Baughman, R. \& Priya, S. [2012]. Hydrogen-fuel-powered bell segments of biomimetic jellyfish, Smart Materials 
and Structures 21(4): 045013. URL: http://stacks.iop.org/0964-1726/21/i=4/a=045013?key= crossref.fb72cd34852f3dfd4f1efbce ff7c630b

[69] Tanaka, K. [1986]. A thermomechanical sketch of shape memory effect: one-dimensional tensile behavior, Res Mechanica 2(3): 59-72.

URL: http://www.sciencedirect.com/science/article/pii/0749641986900161

[70] Teh, Y. H. [2003]. A Control System For Achieving Rapid Controlled Motions From Shape Memory Alloy (SMA) Actuator Wires, PhD thesis, Final Year Project, Australian National University.

[71] Teh, Y. H. [2008]. Fast, Accurate Force and Position Control of Shape Memory Alloy Actuators, $\mathrm{PhD}$ thesis, The Australian National University.

[72] Teh, Y. H. \& Featherstone, R. [2004a]. A new control system for fast motion control of SMA actuator wires, The 1st International Symposium on .

URL: http://axiom.anu.edu.au/roy/SMA/smart04paper.pdf

[73] Teh, Y. H. \& Featherstone, R. [2004b]. Experiments on the Audio Frequency Response of Shape Memory Alloy Actuators, Information Sciences .

[74] Teh, Y. H. \& Featherstone, R. [2004c]. Experiments on the Performance of a 2-DOF Pantograph Robot Actuated by Shape Memory Alloy Wires, Information Sciences pp. 1-8.

[75] Teh, Y. H. \& Featherstone, R. [2007]. Accurate Force Control and Motion Disturbance Rejection for Shape Memory Alloy Actuators, Information Sciences (April): 10-14.

URL: http://ieeexplore.ieee.org/lpdocs/epic03/wrapper.htm?arnumber $=4209783$

[76] Troisfontaine, N., Bidaud, P. \& Dario, P. [1997]. Control experiments on two SMA based micro-actuators, The Fifth International Symposium on Experimental Robotics $V$ Barcelona Spain pp. 490-500.

[77] Villanueva, A., Smith, C. \& Priya, S. [2011]. A biomimetic robotic jellyfish (Robojelly) actuated by shape memory alloy composite actuators., Bioinspiration $\mathcal{E}$ biomimetics 6(3): 036004.

URL: http://www.ncbi.nlm.nih.gov/pubmed/21852714

http://stacks.iop.org/1748-3190/6/i=3/a =036004

[78] Wang, Z., Hang, G., Li, J., Wang, Y. \& Xiao, K. [2008]. A micro-robot fish with embedded SMA wire actuated flexible biomimetic fin, Sensors and Actuators A: Physical 144(2): 354-360.

URL: http://linkinghub.elsevier.com/retrieve/pii/S0924424708001283

[79] Wang, Z., Wang, Y., Li, J. \& Hang, G. [2009]. A micro biomimetic manta ray robot fish actuated by SMA, 2009 IEEE International Conference on Robotics and Biomimetics (ROBIO), IEEE, pp. 1809-1813.

URL: http://ieeexplore.ieee.org/lpdocs/epic03/wrapper.htm?arnumber=5420423

[80] Waram, T. [1993]. Actuator Design Using Shape Memory Alloys, T.C. Waram.

URL: http://books.google.es/books?id=fjZBAgAACAAJ

[81] Westphal, A., Rulkov, N. F., Ayers, J., Brady, D. \& Hunt, M. [2011]. Controlling a lamprey-based robot with an electronic nervous system, Smart Structures and Systems 8(1): 39-52.

[82] Yang, Y., Ye, X. \& Guo, S. [2007]. A New Type of Jellyfish-Like Microrobot, 2007 IEEE International Conference on Integration Technology, number 2006, IEEE, pp. 673-678. URL: http: //ieeexplore.ieee.org/lpdocs/epic03/wrapper.htm?arnumber $=4290404$ http://ieeexplore.ieee.org/xpls/abs_all.jsp?arnumber $=4290404$ 
[83] Zhang, Y., He, J. \& Yang, J. [2006]. Initial research on development of a flexible pectoral fin using Shape Memory Alloy, Automation, Proceedings of pp. 255-260.

URL: $h t t p: / / i e e e x p l o r e . i e e e . o r g / x p l s / a b s \_a l l . j s p ?$ arnumber $=4026090$

[84] Zhong, Z. W. \& Yeong, C. K. [2006]. Development of a gripper using SMA wire, Sensors and Actuators A: Physical 126(2): 375-381.

URL: http://linkinghub.elsevier.com/retrieve/pii/S0924424705005935 